\title{
Insulin production from hiPSC-derived pancreatic cells in a novel wicking matrix bioreactor
}

\author{
Nooshin Amini ${ }^{1}$, Janet Paluh ${ }^{1}$, Yubing Xie ${ }^{1}$, Vinit Saxena ${ }^{2}$, and Susan T. Sharfstein ${ }^{1}$ \\ ${ }^{1}$ SUNY Polytechnic Institute \\ ${ }^{2}$ Sepragen Corporation
}

May 5, 2020

\begin{abstract}
Clinical use of pancreatic beta islets for regenerative medicine applications requires mass production of functional cells. Current technologies are insufficient for large-scale production in a cost-efficient manner. Here, we evaluate advantages of a porous cellulose scaffold and demonstrate scale-up to a wicking-matrix bioreactor as a platform for culture of human endocrine cells. Scaffold modifications were evaluated in a multi-well platform to find the optimum surface condition for pancreatic cell expansion followed by bioreactor culture to confirm suitability. Preceding scale-up, cell morphology, viability and proliferation of primary pancreatic cells were evaluated. Two optimal surface modifications were chosen and evaluated further for insulin secretion, cell morphology and viable cell density for human induced pluripotent stem cell-derived pancreatic cells at different stages of differentiation. Scale-up was accomplished with uncoated, amine-modified cellulose in a miniature bioreactor, and insulin secretion and cell metabolic profiles were determined for 13 days. We achieved 10-fold cell expansion in the bioreactor along with a significant increase in insulin secretion compared with cultures on tissue-culture plastic. Our findings define a new method for expansion of pancreatic cells on wicking-matrix cellulose platform to advance cell therapy biomanufacturing for diabetes.
\end{abstract}

\section{INTRODUCTION}

Diabetes mellitus is one of the leading causes of death, and according to the International Diabetes Federation (IDF), more than 420 million individuals have been diagnosed with diabetes worldwide. Diabetes, or elevated blood sugar, can lead to long-term complications including cardiovascular diseases and damage to the retina, kidney and nerves (Okere 2016, Kieffer 2018). There are two types of diabetes mellitus. In type 1 diabetes, an autoimmune disorder, insulin-producing beta cells are attacked by the immune system, while in type 2 diabetes, insufficient insulin is produced by the beta cells and/or other cells exhibit insulin resistance (Wang 2015). Current treatments are insulin injection and islet transplantation. Insulin injection provides a temporary solution, with risks of inappropriate pre-determined dosing and dependence on external insulin. Islet transplantation remains the only permanent treatment; however, it is complicated by the shortage of donors, required immunosuppression, and risk of tissue rejection. Due to these limitations, a new source of beta cells for type 1 diabetes patients is needed (Vieira 2016, Jiang 2017), which will require advances in human stem cell technologies and biomanufacturing platforms.

Stem cells offer opportunities for patient-specific cell therapies, and induced pluripotent stem cells (iPSC) are considered an attractive replacement for organ transplantation. However, generation and scale-up of patient-specific cells using current protocols is time consuming and costly (Wilmut 2015, Herberts 2011), and whether allogenic cell transplantation provides sufficient immune match compared with autologous cell transplantation is not yet resolved (Millman 2017). Induced pluripotent stem cells, embryonic stem cells, and multipotent stem cells, including bone-marrow mesenchymal stem cells have been successfully differentiated to insulin-producing beta cells in culture (Kieffer, 2016, Jacobson 2017). In order to prevent immune responses to transplanted pancreatic cells, macro- or micro-encapsulation devices have been studied 
(Shultz 2015, Vegas 2016, Bruin 2013). A Phase I clinical trial of a macro-carrier by Viacyte was successfully completed, and the macro-carrier is currently in Phase II clinical trials (https://clinicaltrials.gov identifier: NCT02239354).

Strategies for moving from bench to clinic require mass production of cells either in 2D cell factory cultures as currently performed by Viacyte (Schultz 2015), or in 3D bioreactors, which offer different limitations and advantages. Limited surface area in 2D cultures as well as limited recapitulation of the native organ 3D environment make 2D cultures impractical for scale-up (Kropp 2017). The advantage of 3D culture systems is that cells tend to aggregate into three-dimensional tissue structures that exhibit more natural functional behavior than conventional 2D culture (Modulevsky 2014). Both static and dynamic 3D platforms have been investigated, with the idea that a dynamic system is better able to handle transport of metabolites in high density cell systems than the static one (Kempf 2016). However, dynamic systems, such as stirred and rotating bioreactors, create additional complications of induced shear stress, loss of cells during media changes, non-adaptability of some cell types to suspension culture, cell retrieval and insufficient oxygenation, especially in the case of larger cell aggregates. These limitations can result in cell death and lower the cell yield in these system (Kempf 2016, Kropp 2017). Therefore, an alternative, static, wicking-matrix bioreactor which provides a thin film of medium dripped onto cells on the scaffold offers advantages for 3D culture such as improved oxygen transfer without the detrimental properties of dynamic systems.

Differentiation of human stem cells to insulin-producing pancreatic cells has been performed on synthetic 3D scaffolds including Activin A-grafted gelatin-poly(lactide-co-glycolide) nanoparticle scaffolds, poly(lactideco-glycolide) microporous scaffolds layered with Exendin-4, polyvinyl alcohol scaffolds, and polyether sulfone nanofibrous scaffolds (Kuo 2017, Kasputis 2018, Enderami 2018, Nassiri-Mansour 2018). Synthetic scaffolds offer control over mechanical properties and reproducibility; however, expensive manufacturing techniques and challenges in manipulating surface chemistry to enhance biocompatibility and cell adhesion reduce desirability (Dhandayuthapani 2011, Gervaso 2013).

Cellulose is a naturally abundant, FDA-approved polymer used frequently in biomedical applications including wound dressings and bone tissue engineering (de Oliveira Barud 2016). Cellulose is economical, biocompatible and is readily modifiable to match the desired mechanical and chemical properties (Courtenay 2018). To optimize cell-scaffold functional interactions, we examined six surface modifications of the cellulose scaffold in a multi-well plate assay before scale-up (Figure 1A and Figure 1C). We chose two chemical surface modification approaches. One is the commonly used amine-modification to provide positively charged functional groups for cell binding (Richbourg 2019, Courtenay 2017). The other is a simple $\mathrm{NaOH}$-treatment, which has been shown to enhance surface roughness, hydrophilicity and cell attachment (Chen 2007, Park 2007, Park 2014, Bosworth 2019). We further evaluated whether gelatin-coating enhanced cell attachment cellulose matrices since gelatin (denatured collagen) generally can support cell attachment and growth (Davidenko, 2016). Commercial and in-house hiPSC-derived pancreatic cells expressing NK6 Homeobox 1 (NKX6-1) $)^{+}$pancreatic and duodenal homeobox 1 (PDX-1) ${ }^{+}$were seeded onto scaffolds and evaluated.

Upon determining an optimal scaffold chemistry, we further demonstrated use of a cost-effective, wickingmatrix bioreactor, fabricated by Sepragen Corporation for scale-up biomanufacturing of hiPSC-derived pancreatic cells. The bioreactor consists of a porous amine-modified cellulose scaffold with 20 to $50-\mu$ m wide fibers (Figure 1B) in a sterile chamber with independent air and media inlets and a waste removal outlet (Figure 1D). This system provides a thin film of fresh media on the cell-seeded scaffold and a continuous oxygen environment and liquid waste removal. Our comprehensive analysis includes insulin production, viability, morphology and functionality of cells on scaffolds in culture dishes and bioreactors. Within the scaled-up wicking matrix-bioreactor, we achieved 10-fold expansion and insulin production on amine-modified cellulose scaffolds. Our findings indicate the potential of amine-modified cellulose for future biomanufacturing processes for cell-based diabetic therapies.

\section{MATERIALS AND METHODS}




\section{Scaffold Modification}

Wicking cellulose scaffolds were manufactured by a proprietary "slit and expanded" chemical/mechanical process causing formation of a honeycomb structure with simultaneous heightened airflow and liquid wicking. Amine-modified cellulose scaffolds were manufactured by derivatizing the unmodified cellulose with sodium (meta) periodate followed by a solution of ethylenediamine dihydrochloride, borax and sodium borohydride. Hydroxyl-modified scaffolds were prepared by immersing unmodified scaffolds in $1 \% \mathrm{NaOH}$ solution for 1 hour at $100^{\circ} \mathrm{C}$, followed by 3 washes in each distilled water and phosphate buffered saline (PBS) and air drying overnight. All three scaffolds, unmodified (U), amine-modified (A) and $\mathrm{NaOH}$-modified (N) were also immersed in $1 \%$ gelatin solution for 2 hours in room temperature, followed by air drying overnight to yield UG, AG and NG scaffolds, respectively.

\section{Cell Culture}

hiPSC-derived pancreatic cells were generated following an established stepwise protocol. Briefly, hiPSCs (F3.5.2, Chang 2015) were cultured in mTesr-1 (STEMCELL Technologies) in Matrigel-coated 6-well plates to reach $80 \%$ confluence. When confluent, cells were incubated with Accutase (STEMCELL Technologies) for 5 minutes to detach and pipetted to form single cells. Single cells were centrifuged at $300 \mathrm{x} g$ for 5 minutes; the supernatant was removed, and cells were loaded into custom-made polydimethylsiloxane microarrays prepared by soft lithography (Tomov 2015) with 500- $\mu$ m wells to form embryoid bodies (EB). After four days, EBs were transferred to Matrigel-coated petri dishes and differentiation initiated. Approximately 30 EBs were plated in each well of a 6-well plate. From Day 1 to Day 12 of differentiation, Kroon's protocol (Kroon 2008) was followed to generate endocrine precursors. On day 12, Millman's protocol (Millman 2016) was initiated to further differentiate the cells to mature beta cells.

A frozen vial of primary pancreatic cells was purchased from Celprogen and cultured on pre-coated flasks (Celprogen) according to manufacturer's protocol.

\section{Seeding NKX6-1 ${ }^{+} /$PDX-1 ${ }^{+}$}

\section{Pancreatic Cells on Scaffolds}

\section{Scaffold preparation}

Uniform scaffold samples from all scaffold modifications were prepared using a rectangular punch $(0.5 \times 0.2$ $\mathrm{cm}^{2}$ ) and autoclaved. On the day of the experiment, scaffolds were transferred to 96-well plates and 100 $\mu l$ pancreatic islets of Langerhans cell culture complete growth media (Celprogen) was added to each well. Scaffolds were incubated for 2 hours at $37^{\circ} \mathrm{C}$. Culture media was removed before seeding the cells.

\section{Culture of primary pancreatic cells}

Primary pancreatic cells were removed from culture flasks by incubation with trypsin-EDTA (0.25\%) for 5 minutes. Cells were centrifuged at $100 \mathrm{x}$ g for 5 minutes; the supernatant was removed, and $10 \mu \mathrm{l}$ of cell suspension containing 20,000 cells was added to each scaffold. After 4 hours, $100 \mu$ l culture media was added to each well. To maintain pancreatic cells on scaffolds, medium was changed every other day for 10 days. Six replicate cultures of all six scaffold modifications were performed for each experiment.

\section{Culture of hiPSC-derived pancreatic cells}

hiPSC-derived pancreatic cells (day 12 of differentiation) were seeded on scaffolds with two different modifications: amine-modified and gelatin-coated, $\mathrm{NaOH}$-modified. Cells were seeded either as single cells or as aggregates. For seeding single cells, the aforementioned method was followed. To seed the aggregates, cell islets with an average of 4000 cells were detached manually from wells of a 6 -well plate by a $10 \mu \mathrm{l}$ pipette tip under microscopic observation and were transferred to a $15 \mathrm{ml}$ centrifuge tube followed by gravitational settling. After 10 minutes at $37^{\circ} \mathrm{C}$, the supernatant was removed, and aggregates were resuspended in 360 $\mu \mathrm{l}$ of stage-four medium (DMEM/F12, B27, p/s). $10 \mu \mathrm{l}$ of cell suspension, containing 5 aggregates was added 
to each prepared scaffold. The same differentiation protocol was followed for cells grown on scaffolds as in dishes.

\section{MTT (3-(4,5-Dimethyl-2-thiazolyl)-2,5-diphenyltetrazolium bromide) Viable Cell Assay}

After seeding primary pancreatic cells and hiPSC-derived pancreatic cells on scaffolds, 1, 3, 5 and 10 days after seeding, the viable cell density on each scaffold was assessed by MTT assay (Invitrogen). Six replicates were performed for each scaffold modification. Following the manufacturer's instructions, scaffold culture media of cells grown on scaffolds were changed to fresh media and $10 \mu$ l reconstituted MTT reagent was added to each well of the 96-well plate. Scaffolds were incubated overnight. The next day, MTT solution was aspirated and $100 \mu \mathrm{l}$ solubilization solution was added to scaffolds. Solubilized MTT solutions were transferred to clean 96-well plates and solution absorbance was measured at $570 \mathrm{~nm}$ on a Tecan infinite 200 microplate reader.

\section{Scanning Electron Microscopy}

Scaffolds were fixed in $100 \mu$ l fixing solution $(2.5 \mathrm{M}$ glutaraldehyde, $0.2 \mathrm{M}$ phosphate buffer, $1 \mathrm{mM}$ sucrose solution) for 2 hours at room temperature, washed three times with washing buffer (0.05 M sucrose, 0.05 M phosphate buffer, pH 7.2) for 10 minutes. Samples were dried by liquid displacement using an ethanol: water gradient $(25 \%, 50 \%, 70 \%, 80 \%, 95 \%, 100 \%)$ and then ethanol: hexamethyldisilazane $(3: 1,1: 1,1: 3)$. Finally, samples were air dried overnight. Dried samples were sputter coated with gold/palladium, mounted and imaged on a LEO 1550 scanning electron microscope.

\section{Insulin Secretion ELISA}

Insulin secretion from hiPSC-derived pancreatic cells cultured on scaffolds was assessed by ultrasensitive insulin ELISA kit (ALPCO 80-INSHUU-E01.1) at stages 4, 5 and 6 of differentiation (days 1,5 and 10 post seeding in 96-well plates and every other day in bioreactors). Following the manufacturer's instructions, cellculture supernatants, standard solutions, and controls were added to anti-insulin pre-coated microplate wells and incubated with detection antibody at room temperature with shaking for 1 hour. Subsequently, the microplate was washed six times with provided wash buffer and incubated with 3,3',5,5'-Tetramethylbenzidine substrate for 30 minutes with shaking. After addition of the stop solution, the absorbance of each sample was measured at $450 \mathrm{~nm}$.

\section{Bioreactor Inoculation}

A miniature (100-ml working volume) wicking bioreactor was sterilized by ethylene oxide gas. The scaffold was pre-wetted with $100 \mathrm{ml}$ DMEM/F12 medium overnight. The next day, hiPSC-derived pancreatic cells were trypsinized, and resuspended in $50 \mathrm{ml}$ stage 4 culture medium. Cells were inoculated into the bioreactor by adding the cells to the feed bottle and recirculating the medium. Within 12 hours, no free-floating cells were seen in the feed bottle. Cell culture media were changed every other day, following the differentiation protocol. Upon completion of the bioreactor experiment, cells were removed by placing the scaffold in $20 \mathrm{ml}$ of Dulbecco's modified Eagle's medium containing 0.5\% Trypsin with EDTA and sonicating for 3 hours at $40 \mathrm{~Hz}$ using an ultrasonic bath. Cell density of the released cells in the medium was assessed by two methods, Vi-cell counter and hemocytometer.

\section{Metabolic Profile}

Bioreactor culture medium samples were taken every other day while changing the medium. One ml of supernatant was used to measure glucose consumption and lactate production using a YSI 2950 biochemistry analyzer.

\section{Immunocytochemistry}

Cells were fixed with $10 \%$ formalin for 20 minutes at room temperature, washed with $0.01 \mathrm{M}$ phosphate buffer ( $\mathrm{pH}$ 7.4) three times, permeabilized in phosphate buffer with $0.5 \%$ Triton X-100 for 10 minutes at room temperature, washed three times with PBS, blocked with blocking buffer (BSA + Triton X-100) for 
1 hour at room temperature, and labeled with 1:1000 dilution of primary antibodies (Sox9, Sox17, FoxA2, Brachyury, PDX-1, NGN3, NeuroD1, MafA, NKX2-2, NKX6-1, insulin) in blocking buffer overnight at $4^{\circ}$ C. Samples were then incubated for an hour with 1:1000 donkey anti-mouse IgG labeled with Alexa Fluor 488 and/or donkey anti-goat, donkey anti-rabbit or donkey-anti rat IgG labeled with Alexa Fluor 594 (Thermofisher) as appropriate for the primary antibody. Sampled were mounted with Prolong diamond antifade mountant (Thermofisher) and imaged on a Zeiss Axio observer Z1 fluorescence microscope.

\section{Statistical Analysis}

One-way and two-way ANOVA were performed using GraphPad Prism for Windows version 8.3.0, www.graphpad.com.

\section{RESULTS}

\section{Expansion of Primary Pancreatic Cells on the Cellulose Scaffold is Affected by Scaffold Surface Modification}

To determine optimal conditions for pancreatic cell growth on the porous cellulose scaffold, cellulose pieces (5 $\mathrm{mm} \times 3 \mathrm{~mm} \times 2 \mathrm{~mm}$ ) from wicking matrix material subjected to various treatments were evaluated in a multi-well platform (Figure 1C) for cell attachment/retention, viability, cell penetration into the scaffold, morphology, and functionality of cells on the scaffold. Purchased human primary pancreatic cells (Islets of Langerhans) were cultured and expanded in coated flasks to reach the desired number for seeding onto scaffold and validated by immunocytochemistry for relevant intracellular pancreatic biomarkers, including PDX-1, NeuroD1, NGN3 and NKX6-1 (Figure 2A). These pancreatic cells, referred to as NKX6-1+ $/ P D X-1^{+}$, were collected by trypsinization, and 20,000 cells were seeded onto cellulose scaffolds that were either unmodified, amine- or $\mathrm{NaOH}$-modified, with or without gelatin coating. Cells were maintained for 10 days on these scaffolds and evaluated at days 1, 3, 5 and 10 by MTT assay (Figure 2B) and scanning electron microscopy (Figure 3). The time course of cell growth on the six different cellulose scaffolds was measured by MTT assay. On day 1 , viable cells were observed to attach efficiently to all six scaffold modifications (Figure 2B, Figure 3). On day 3, amine-modified (A), amine-modified, gelatin-coated (AG), and $\mathrm{NaOH}$-modified, gelatin-coated (NG) scaffolds showed significantly greater viable cell densities than the other scaffolds (one-way ANOVA, $\mathrm{p}<0.001$ ), while on day 5 , the unmodified, non-coated scaffolds showed lower viable cell densities than any of the other scaffolds (one-way ANOVA, p < 0.001). Interestingly, by day 10, cells grown on the unmodified, uncoated scaffolds had recovered, and no statistically significant differences in cell density between any of the modifications were observed based on MTT assay, though qualitative differences were observed based on SEM imaging as described below. The MTT assay data were further used to calculate the expansion of pancreatic progenitors under the six modification conditions to find the optimum surface conditions for attachment and growth of pancreatic progenitors (Table 1). The MTT assay results indicate that the NKX6-1+ $/ P D X$ $1^{+}$pancreatic progenitor cells attached and expanded to some degree in all six conditions. The largest expansion achieved was 6.6-fold on the gelatin-coated, $\mathrm{NaOH}$-modified (NG) scaffolds; whereas only 3.8-fold expansion was observed on similarly modified $\mathrm{NaOH}$ scaffolds lacking gelatin coating $(\mathrm{N})$. Gelatin coating was not always required, since unmodified (U) and amine-modified (A) scaffolds without gelatin coating had the next highest expansion with 5.6- and 5.4-fold expansion respectively. Based on our observations, uncoated, amine-modified (A) scaffolds and gelatin-coated, $\mathrm{NaOH}$-modified (NG) scaffolds provided better surface attachment and higher growth rates for primary pancreatic cells (Figure 2B) and were selected for further studies.

\section{High Resolution Imaging of Primary Pancreatic Cell Attachment and Expansion on Cellulose Scaffolds}

Due to the non-transparent nature of the cellulose scaffold, we examined cell attachment and extent of coverage on the scaffolds by SEM on days 1, 3, 5 and 10 after seeding (Figure 3). On day one, for all surface modifications, cells were distributed throughout the scaffolds. By day three, cells produced visible cytoplasmic extensions and were distributed more widely on the scaffolds. We observed a clear preference for non-coated unmodified (U) and amine-modified (A) and gelatin-coated, NaOH-modified (NG) surfaces 
compared with other conditions (Supplemental Figure 1). Significant cell expansion was observed by day five, along with cell migration into the scaffold interior pockets. Cell aggregates were also observed as well as extracellular matrix (ECM) on the scaffolds as shown in the images. These results were consistent with our MTT-assay quantification of viable cells over time under each condition. Cell aggregates were seen on uncoated, unmodified (U) and amine-modified (A) scaffolds more than on other surface conditions, which show a more extensive cell distribution (Supplemental Figure 1).

\section{hiPSC-Derived NKX6-1 + PDX-1 ${ }^{+}$Pancreatic Cells Bound to Both NaOH- and Amine- modified Scaffolds}

hiPSC-derived pancreatic cells were generated and matured to beta cells by combining two existing protocols (Kroon 2008, Millman 2016) and validated by immunocytochemical analysis of pancreatic markers (Figure 4) similar to previous experiments with primary pancreatic cells. On day 12 of differentiation, cells were detached and seeded onto cellulose scaffolds as single cells or as small aggregates. Based on the results of the MTT assay and SEM analysis using primary pancreatic cells, two conditions, uncoated amine-modified (A) scaffolds and gelatin-coated, $\mathrm{NaOH}$-modified (NG) scaffolds were evaluated. Similar to previous experiments, 20,000 hiPSC-derived endocrine precursors were seeded on scaffolds and differentiation was continued in stage 5 and 6 differentiation media (Millman 2016) for 10-12 additional days (10 days for aggregates, 12 days for single cells), which should result in immature beta cells expressing markers such as insulin, NKX6-1, PDX-1 and MAF BZIP transcription factor A (MafA). The viable cell density was analyzed by MTT assay on days 1, 5 and 10/12, and cells successfully attached and proliferated on both surfaces (Figure 5A and Figure 5B). In comparison with single cells, the seeded aggregates had higher initial viable cell density; however, the viable cell density declined over time for cells on both surface conditions, with a very significant decrease on the gelatin-coated, $\mathrm{NaOH}$-modified (NG) scaffolds on day 5 , which reduced the number of viable cells to less than half of the initial number (Figure 5B). In contrast, the viable cell density for single cells on the uncoated amine-modified (A) scaffolds increased linearly over time. Even though single cells on the gelatincoated, $\mathrm{NaOH}$-modified (NG) scaffolds demonstrated slightly higher viable cell density than single cells on the amine-modified scaffolds, the viable cell number dropped suddenly on day 5 , similarly to aggregates. To determine if cell viability was being affected or if cells were detaching from the scaffold surface, we used SEM to assess the attachment and morphology of the differentiating hiPSC-derived pancreatic cells on the scaffolds on days 1, 5 and 12. During differentiation, we observed a significant amount of ECM produced on uncoated amine-modified scaffolds loaded with single cells and aggregates (Figure 5E). In contrast, ECM deposition was lower on gelatin-coated, $\mathrm{NaOH}$-modified (NG) scaffolds seeded with aggregates and single cells (Figure 5F). Images of aggregates on scaffolds from day 12, entering stage 6 (immature beta cells), reveal an almost empty scaffold, in agreement with MTT data showing a decrease in number of viable cells (Figure 5A). In both single cells and aggregates assays, uncoated amine-modified surfaces showed more uniform cell attachment and growth compared to gelatin-coated, NaOH-modified surfaces, and therefore, proved to be a better candidate for pancreatic cell culture.

\section{Scaffold-Supported hiPSC-Derived NKX6-1+/PDX-1 ${ }^{+}$Pancreatic Cells Secrete Insulin}

Insulin release by pancreatic cells on uncoated amine-modified (A) scaffolds and gelatin-coated, NaOHmodified (NG) scaffolds was quantified by ELISA on days 1, 5 and 10/12. Single cells on amine-modified (A) scaffolds produced the highest amount of insulin on day 1 (Figure 5C); however, insulin production declined on days 5 and 12, similar to single cells on gelatin-coated, $\mathrm{NaOH}$-modified (NG) scaffolds (Figure $5 \mathrm{D})$. The decrease in insulin production was consistent with loss of cells from the scaffolds. It is unclear if the remaining cells attached to the scaffolds exhibited any change in insulin production. Cell aggregates on both scaffold modifications had a very low insulin release at day 1 . Even though insulin release increased by day 5 on both surfaces, it was still approximately half of the insulin released from single cells. On both surface modifications, insulin concentration surpassed that produced by a similar number of pancreatic cells cultured in a dish (data not shown). Based on ELISA results, single cells seeded on scaffolds secreted higher amounts of insulin than aggregates cultured on scaffolds, which in turn secreted more insulin than cells cultured on tissue culture dishes, with the highest amount produced by single cells on uncoated amine-modified (A) 
scaffolds.

\section{Insulin Secretion by Scaffold-Attached hiPSC-Derived NKX6-1 ${ }^{+} /$PDX-1 ${ }^{+}$Pancreatic Cells Increases with Integration into the Bioreactor}

A miniature bioreactor was manufactured with the uncoated amine-modified (A) wicking matrix scaffold as that scaffold modification demonstrated the greatest potential for cell expansion and insulin production in our previous experiments. The bioreactor scaffold was pre-wetted with medium and then seeded with 5 x $10^{6}$ or $1 \times 10^{7}$ hiPSC-derived pancreatic cells (Figure 6A). Insulin release and metabolite profiles were determined from assays of the culture medium. At the conclusion of experiments, MTT was added to the bioreactor to visualize viable cells (Figure 6B), and SEM visualization of cell attachment and distribution throughout the scaffold from the bioreactor was performed (Figure 6C).

Insulin ELISA of bioreactor samples from $1 \times 10^{7}$ seeded cells showed an insulin production greater than $0.28 \mu \mathrm{g}$ on day 2 of the bioreactor run. Insulin production was again evaluated at day 4, 6, 8, 10 and 13 . Insulin production on day 6 increased to $0.3 \mu \mathrm{g}$ and remained high through the last examined time point, day 13 (Figure 6D). When fewer cells were seeded $\left(5 \times 10^{6}\right)$ onto the bioreactor, an initial lag in insulin production was observed, but by days 7 to 13 higher insulin output was achieved, although slightly reduced versus seeding with $1 \times 10^{7}$ cells.

In addition to insulin production, we evaluated the metabolic profile of cells seeded at the $1 \times 10^{7}$ cell density for glucose and lactate production that are typically good indicators of process robustness. The metabolic profile of cells was determined using a YSI biochemical analyzer (Figure 6E). Glucose consumption in the bioreactor seeded with $1 \times 10^{7}$ cells increased $\sim$-fold between day 2 and day 6 , after which the glucose consumption decreased $\sim 15 \%$ between day 6 and 8 and another $10 \%$ by the end of experiment. Lactate production was much lower than glucose consumption and showed an opposite trend from glucose consumption, with a substantial drop in lactate production between day 2 and 8 , followed by an increase until the end of the culture. The relatively high glucose consumption throughout the experiment is indicative of sustained metabolic activity of the cells in the bioreactor over 13 days. These results show cells in the bioreactor were able to attach to and grow on an amine-modified scaffold, maintain a steady metabolic activity, and produce high amounts of insulin for 13 days. A bioreactor inoculated with $5 \times 10^{6}$ cells was disassembled after 14 days and cells were harvested from the amine-modified scaffold by trypsinization and sonication; $50 \times 10^{6}$ cells were counted, demonstrating ${ }^{\sim} 10$-fold expansion of differentiating pancreatic cells in bioreactor.

\section{DISCUSSION}

The need for innovative biomanufacturing platforms that sustain cell viability and functionality are critical to broadly advancing cell therapies. In this study, we demonstrated production of insulin from metabolically competent pancreatic cells seeded onto a wicking matrix cellulose scaffold. We tested multiple surfacemodification conditions for cell attachment and expansion in a multi-well test platform and small-scale bioreactor. Cells seeded onto the wicking matrix cellulose scaffold in the bioreactor exhibited greater expansion (up to 10-fold) and increased insulin secretion, when compared with traditional 2D cultures. Metabolic profiling indicates that the oxygenation and physiological conditions in bioreactor were favorable. This biomanufacturing platform provides an efficient 3D microenvironment for pancreatic cells to support mass production of these insulin producing cells for potential use in cell therapy to treat Type 1 diabetes patients. The ability to use human induced pluripotent stem cell-derived pancreatic cells also opens up the possibility of patient-specific treatments.

Multiple surface modifications enabled attachment and growth of primary pancreatic cells as evaluated by MTT metabolic assay and scanning electron microscopy. Before seeding cells, a fraction of the culture was validated in parallel for relevant biomarker expression by immunocytochemistry, including PDX-1, NGN3, NKX6-1, NeuroD1 and insulin, which are indicators of pancreatic lineage-committed cells (Takahashi 2016, Kieffer 2014, Benthuysen 2016). Pancreatic cells seeded at a density of 20,000 cells/well on various modified scaffolds in a multi-well platform were able to attach similarly to cellulose with all six surface modifications; 
however, their expansion varied considerably. Growth on uncoated, amine-modified cellulose (A) and gelatincoated, $\mathrm{NaOH}$-modified cellulose (NG) scaffolds provided the most favorable surface conditions for expansion of cells with 5 to 6 -fold expansion. By high resolution SEM imaging of cells on day 1 and day 3, we observed spreading of cells on these scaffolds with visible lamellipodia formation. As discussed by several authors (Banik 2015, Le 2013, Krishnamurthy 2009), surface topography of various scaffolds contributes to cell attachment and can impact long-term viability and differentiation of cells. The cellulose scaffold supports cell spreading and retention of phenotypic function. Seeded pancreatic cells displayed an affinity towards all modified surfaces of the porous cellulose scaffold that was apparent by formation of structures that resemble lamellipodia and production of a film of extracellular matrix. In addition, cells were able to form larger aggregates over 5-10 days, consistent with survival and proliferation seen in other studies (Kim 2016, Salvatori 2014). Between day 1 and day 10, sustained culture on the amine-modified scaffolds resulted in better expansion, based on a greater distribution of cell clusters throughout the scaffold.

Our analysis revealed that the uncoated, amine-modified (A) and gelatin-coated, NaOH-modified (NG) scaffolds were most favorable, and these were chosen for additional analysis using hiPSC-derived pancreatic cells. Viability, morphology and insulin secretion of hiPSC-derived pancreatic cells on these two scaffold modifications were examined. The human iPSC-derived endocrine cells were generated by multiple stages of differentiation. In the first stage of pancreatic differentiation, expression of definitive endoderm biomarkers, such as Sox 17 and Brachyury increase while pluripotency markers decrease. Moving developmentally toward posterior foregut, pancreatic biomarkers such as PDX-1 appear. Following commitment to the pancreatic lineage, expression of biomarkers such as NKX2-2, NKX6-1, Neurogenin-3 and insulin increase (D'Amour 2006, Rezania 2012, Russ 2015). From committed pancreatic cells, the hormone-releasing beta cells arise; however, these cells are initially immature, and the amount of insulin released in response to changes in glucose level is low. Further differentiation to mature, beta islet cells is facilitated by addition of Triiodothyronine (T3) which is a Thyroid receptor agonist, with observed upregulation of MafA in cultures (Aguayo-Mazzucato 2013, Millman 2016). We tested hiPSC-derived pancreatic progenitors, NKX6-1+/PDX-1+ on our uncoated, amine-modified (A) and gelatin-coated, $\mathrm{NaOH}$-modified (NG) cellulose scaffolds for 10 days as single cells and as lightly dissociated cell aggregates. By MTT assay, we observed a predictable trend of decreased cell proliferation as cells underwent differentiation on amine-modified scaffolds from seeded aggregated cells. We observed that cell aggregates had higher viable cell density on amine-modified (A) scaffolds compared to gelatin-coated, $\mathrm{NaOH}$-modified (NG) scaffolds on all days of testing. Viable cell density from single-seeded cells on amine-modified scaffolds (A) increased linearly throughout the experiment. However, single cells seeded on gelatin-coated, $\mathrm{NaOH}$-modified scaffolds (NG) showed a significant decrease in viable cells on day 5. These findings suggest that the differentiating seeded pancreatic cells were more compatible with the amine-modified scaffolds. Images taken by SEM support this observation. SEM images acquired on day 5 showed production of significant amount of ECM on the amine-modified scaffolds seeded with cell aggregates. The presence of ECM was also observed on day 5 and day 10 on amine-modified scaffolds seeded with single cells. As noted previously (Hammer 2004, Stendhal 2009, Llacua 2018, Smink 2018), ECM production is a necessary step for support of multiple cell activities on a scaffold including survival and migration, and in some cases, ECM components can integrate into the scaffold structure to enhance cell attachment, differentiation and proliferation for tissue engineering.

A sustained ability to produce insulin is critical to biomanufacturing efforts with pancreatic cells. Insulin secretion was measured for cells grown on both the uncoated, amine-modified (A) scaffold and gelatincoated, NaOH-modified (NG) scaffold, and both demonstrated greater insulin release than was observed from the same cells in 2D culture (data not shown). This analysis demonstrates the enhanced functional potential of chemically modified 3D cellulose scaffolds for supporting hiPSC-derived pancreatic cells and differentiation into mature, functional, insulin-secreting pancreatic cells. The amount of insulin secreted from single cells seeded on both cellulose surface-modified conditions decreased over time, in contrast to cell aggregates seeded, which showed an increase in insulin secretion on day 5. We speculate that the higher insulin secretion and ECM production by cell aggregates on amine-modified scaffolds is due to higher cell density and increased cell-cell contact on the scaffolds. As a follow up, we tested insulin release from 
single cells seeded on the scaffold in the bioreactor at two seeding densities. The hiPSC-derived endocrine precursors were seeded onto the amine-modified wicking matrix scaffold in the bioreactor, and insulin release and metabolic activity of cells were assessed for 13 days. We observed a significant increase in insulin secretion at both cell seeding densities that remained high until the conclusion of the experiment, demonstrating an improvement of cell functionality in this 3D environment. The porous structure of cellulose fibers along with adequate oxygenation and continuous accessibility of all cells to media in the bioreactor appear to meet critical microenvironment needs enabling $\sim$-fold increase in insulin release in the bioreactor. Doubling the cell density enhanced insulin production in the bioreactor, reaching the higher insulin secretion by day 2 rather than day 7. Given that cell seeding density affects differentiation and maturation of pancreatic cells in 2D cultures (Gage 2013), we suspect that delayed insulin production at the lower cell seeding density in the bioreactor is a result of an arrested or slowed maturation process.

Metabolically, glucose consumption and lactate production in the bioreactor were reflective of good cell viability and activity. Low glucose consumption levels, which can be a sign of lowered cell viability, were not observed. Higher inoculation densities $\left(1 \times 10^{7}\right)$ exhibited increased glucose consumption and lactate production compared to lower cell densities (i.e., $5 \times 10^{6}$ cells, Supplemental Figure 2). The relatively low levels of lactate produced in the bioreactor indicate sufficient aeration, as would be expected in the wicking matrix bioreactor with the thin film of liquid. In addition, the low lactate concentration demonstrates a low rate of aerobic glycolysis. Elevated aerobic glycolysis is a hallmark of tumor cells as well as dedifferentiation in culture. In summary, the metabolic profile further supports the design and use of the bioreactor for cell biomanufacturing.

\section{CONCLUSION}

In this study, attachment and expansion of immature and maturing insulin-secreting pancreatic cells on various modifications of the porous cellulose scaffold was examined in a high-throughput, multi-well platform and scaled-up in a wicking matrix bioreactor. Our findings demonstrate the ability of pancreatic progenitors to attach and expand on modified cellulose and further indicate that the amine-modified cellulose scaffold, in particular, can support 10-fold expansion of hiPSC-derived insulin-secreting pancreatic cells in a scaled-up bioreactor while maintaining steady metabolic activity over 13 days. We expect this platform to be useful in future biomanufacturing of pancreatic cells for advancing cell therapies for diabetes patients.

\section{ACKNOWLEDGMENTS}

This work was supported by funding from the National Science Foundation IIP-1722384. The authors would like to thank Andrew Burns for experimental suggestions, Andrew Premo for assisting in MTT assays and Steven Rose for assisting with sample preparations.

\section{CONFLICT OF INTEREST}

Vinit Saxena is President of Sepragen, Inc., a company commercializing the Express Wicking Matrix Bioreactor. Susan Sharfstein was a co-Principal Investigator on an NSF STTR grant with Sepragen.

\section{REFERENCES}

Aguayo-Mazzacuto, C., Zavacki, A.M., Marinelarena, A., Hollisyer-Lock, J., El Khattabi, I. Marsili, A., Weir, G. C., Sharma, A., Larsen, P. R., \& Bonner-Weir, S. (2013). Thyroid hormone promotes postnatal rat pancreatic $\beta$-cell development and glucose-responsive insulin secretion through MAFA. Diabetes , 62 , 1569-1580. Banik, B. L., \& Brown, J. L. (2015). Interaction of responsive/switchable surfaces with cells. In Switchable and Responsive Surfaces and Materials for Biomedical Applications (pp. 189-201). Elsevier Inc. Benthuysen, J. R., Carrano, A. C. $\&$ Sander, M. (2016). Advances in $\beta$ cell replacement and regeneration strategies for treating diabetes.J. Clin. Invest. 126 (10), 3651-3660. Bosworth, L. A., Hu, W., Shi, Y. \& Cartmell, S. H. (2019). Enhancing biocompatibility without compromising material properties: an optimised $\mathrm{NaOH}$ treatment for electrospun polycaprolactone fibers. J of Nanomat, 2019. Bruin, J. E., Rezania, A., Xu, J., Narayan, K., Fox, J. K., O’Neil, J. J. \& Kieffer, T. J. (2013). Maturation 
and function of human embryonic stem cell-derived pancreatic progenitors in macroencapsulation devices following transplant into mice. Diabetologia , 56 , 1987-1998. Chang, E. A., Tomov, M. L., Suhr, S. T., Luo, J., Olmsted, Z. T., Paluh, J. L., Cibelli, J. (2015). Derivation of Ethnically Diverse Human Induced Pluripotent Stem Cell Lines. Sci Rep , 5 , 15234. Chen, F., Lee, C. N. \& Teoh, S. H. (2007). Nanofibrous modification on ultra-thin poly(e-caprolactone) membrane via electrospinning. Mat sci \& Eng C , 27, 325-332. Courtenay, J. C., Sharma, R. I. \& Scott, J. L. (2018) Recent Advances in Modified Cellulose for Tissue Culture Applications. Molecules ,23 , 654-674. Courtenay, J. C., Johns, M. A., Galenbeck, F., Deneke, C., Lanzoni, E. M., Costa, C. A., Scott, J. L. \& Sharma, R. I. (2017). Surface modified cellulose scaffolds for tissue engineering. Cellulose, 24, 253-267. D'Amour, K. A., Bang, A. G., Eliazer, S., Kelly, O. G., Agulnick, A. D., Smart, N. G., Moorman, M. A., Kroon, E., Carpenter, M. K. \& Baetge, E. E. (2006). Production of pancreatic hormone - expressing endocrine cells from human embryonic stem cells. Nat Biotech ,24 (11), 1392-1401. Davidenko, N., Schuster, C. F., Bax, D. V., Farndale, R. W., Hamaia, S., Best, S. M. \& Cameron, R. E. (2016). Evaluation of cell binding to collagen and gelatin: a study of the effect of 2D and 3D architecture and surface chemistry. J Mater Sci: Mater Med , 27, 148-162. De Oliveira Barud, H. G., da Silva, R. R., da Silva Barud, H., Tercjak, A., Gutierrez, J., Lustri, W. R., de Oliveira Junior, O. R., Ribeiro, S. J. L. (2016). A multipurpose natural and renewable polymer in medical applications: bacterial cellulose. Carbohydrate Polymers ,153 , 406-420. Dhandayuthapani, B., Yoshida, Y., Maekawa, T. \& Kumar D. S. (2011). Polymeric scaffolds in tissue engineering application: A review. Int J Polymer Sci, 2011. Enderami, SE., Soleimani, M., Mortazavi, Y., Naderi, S. \& Salimi, A. (2018). Generation of insulin-producing cells from human adipose-derived mesenchymal stem cells on PVA scaffold by optimized differentiation protocol. J Cell phys , 233 (5), 4327-4337. Gervaso, F., Sannino, A. \& Peretti, G. M. (2013). The biomaterialist's task: scaffold biomaterials and fabrication technologies. Joints, 1(3), 130-137. Hammer, E., Parnaud, G., Bosco, D, Perriraz, N., Maedler, K., Donath, M., Rouiller, D. G. \& Halban, P. A. (2004). Extracellular matrix protects pancreatic B-cells against apoptosis. Diabetes ,53 , 2034-2041. Herberts, C.A., Kwa, M. SG. \& Hermsen, H. PH. (2011). Risk factors in the development of stem cell therapy. J. Trans. Med. 9 , 29-43. Jacobson, E. F. \& Tzanakakis, E. S. (2017). Human pluripotent stem cell differentiation to functional pancreatic cells for diabetes therapies: Innovations, challenges and future directions. J. Biol. Eng.,11 , 21-34. Jiang, Y. \& Chen, D. (2017). Stem Cell-based Approach in Diabetes and Pancreatic Cancer Management. Cancer Transl Med , 3 (1), 348. Kaputis, T., Clough, D., Noto, F., Rychel, K., Dye, B. \& Shea, L. D. (2018). Microporous polymer scaffolds for the transplantation of embryonic stem cell derived pancreatic progenitors to a clinically translatable site for treatment of type I diabetes. ASC Biomaterials , 4 , 1770-1778. Kempf, H., Andree, B. \& Zweigerdt, R. (2016). Large-scale production of human pluripotent stem cell derived cardiomyocytes. Adv. Drug Deliv. Rev. 96, 18-30. Kieffer, T. J. (2016). Forum closing in on mass production of mature human beta cells. Cell Stem Cell , 18 , 699-702. Kieffer, T. J., Woltjen, K., Osafune, K., Yabe, D., \& Inagaki, N. (2018). Beta-cell replacement strategies for diabetes. J Diabetes Investig , 9 (3), 457-463. Kim, Y., Kwon, I. K., \& Shin, K. (2016). Extracellular matrix revisited: roles in tissue engineering. Int Neuronal J ,20 (S1), 23-29. Krishnamurthy, M., \& Wang, R. (2009). Integrins and extracellular matrices in pancreatic tissue engineering. Frontier in Biosciences , S1 , 477-491. Kroon, E., Martinson, L. A., Kadoya, K., Bang, A. G., Kelly, O. G., Eliazer, S., Young, H., Richardson, M., Smart, N. G., Cunningham, J., Agulnick, A. D., D'Amour, K. A., Carpenter, M. K. \& Baetge, E. E. (2008). Pancreatic endoderm derived from human embryonic stem cells generates glucose-responsive insulin-secreting cells in vivo. Nat biotech , 26 (4), 443-453. Kropp, C., Massai, D. \& Zweigerdt, R. (2017). Progress and challenges in large-scale expansion of human pluripotent stem cells. Process Biochem. 59 , 244254. Kuo, Y., Liu, Y. \& Rajesh, R. (2017). Pancreatic differentiation of induced pluripotent stem cells in activin A-grafted gelatin-poly(lactide-co-glycolide) nanoparticle scaffolds with induction of LY294002 and retinoic acid. Mat Sci \& Eng C , 77 , 384-393. Le, X., Poinern, G. E. J., 
Ali, N., Berry, C. M., \& Fawcett, D. (2013). Engineering a biocompatible scaffold with either micrometer or nanometer scale surface topography for promoting protein adsorption and cellular response. Int J Biomaterials, 2013. Llacua, L. A., Faas, M. M., de Vos, P. (2018). Extracellular matrix molecules and their potential contribution to the function of transplanted pancreatic islets. Diabetologia, 61 , 1261-1272. Mansour, R. N., Barati, G., Soleimani, M., Ghoraeian, P., Nouri-Aleagha, M., Kehtari, M., Mahboudi, H., Hosseini, F., Hasannia, H., Abazari, M. F. \& Enderami, SE. (2018). Generation of high-yield insulin producing cells from human-induced pluripotent stem cells on polyethersulfone nanofibrous scaffold. Artif Cell Nanomed biotechnol , 46 , 733-739. Millman, J. R., \& Pagliuca, F. W. (2017). Autologous pluripotent stem cell derived $\beta$-like cells for diabetes cellular therapy.Diabetes , 66 , 1111-1120. Millman, J. R., Xie, C., Dervort, A. V., Gürtler, M., Pagliuca, F. W. \& Melton, D. A. (2016). Generation of stem cell-derived b cells from patients with type 1 diabetes. Nat commun , 7, 11463. Modulevsky, D. J., Lefebvre, C., Haase, K., Al-Rekabi, Z. \& Pelling, A. E. (2014). Apple derived cellulose scaffold for 3D mammalian cell culture. Plos one , 9 (5), e97835. Okere, B., Luacaccioni, L., Dominici, M., \& Lughetti, L. (2016). Cell therapies for pancreatic beta-cell replenishment. Italian J Pediatrics, 42, 62-71. Park, J. S., Kim, JM., Lee, S. J., Lee, S. G., Jeong, YK., Kim, S. E. \& Lee, S. E. (2007). Surface hydrolysis of fibrous poly( $\varepsilon$-caprolactone) scaffolds for enhances osteoblast adhesion and proliferation.Macromolecular Research , 15 (5), 424-429. Park, S., Lee, J., Kim, Y., Kim, J., Il, K.K., Lee, J., Kim, W., Kim, H., Kim, M. \& Lee, J. (2014). Effect of sodium hydroxide treatment on scaffold by solid freeform fabrication. Polymer Korea ,38 (6), 815-819. Rezania, A., Bruin, J. E., Riedel, M. J., Mojibian, M., Asadi, A., Xu, J., Gauvin, R., Narayan, K., Karanu, F., O'Neil, J. J., Ao, Z., Warnock, G. L. \& Kieffer, T. J. (2012). Maturation of human embryonic stem cell-derived pancreatic progenitors into functional islets capable of treating pre-existing diabetes in mice. Diabetes , 61 , 2016-2029. Rezania, A., Bruin, J. E., Arora, P., Rubin, A., Batushansky, I., Asadi, A., O’Dwyer, S., Quiskamp, N., Mojibian, M., Albrecht, T., Yang, Y. H. C., Johnson, J. D. \& Kieffer, T. J. (2014). Reversal of diabetes with insulin-producing cells derived in vitro from human pluripotent stem cells. Nat Biotech, 32 (11), 1121-1133. Richbourg, N. R., Peppas, N. A. \& Sikavitsas, V. I. (2019). Tuning the biomimetic behavior of scaffolds for regenerative medicine through surface modifications. J Tissue Eng Regen Med , 13 (8), 1275-1293. Russ, H. A., Parent, A.V., Ringler, J. J., Hennings, T. G., Nair, G. G., Shveyjert, M., Guo, T., Puri, S., Haataja, L., Cirulli, V., Blellock, R., Szot, G. L., Arvan, P. \& Hebrok, M. (2015). Controlled induction of human pancreatic progenitors produces functional beta-like cells in vitro. The EMBO J , 34 , 1759-1772. Salvatori, M., Katari, R., Patel, T., Peloso, A., Mugweru, J., Owusu, K., \& Orlando, G. (2014). Extracellular matrix scaffold technology for bioartificial pancreas engineering. $J$ Diabetes Sci Technol ,8 (1), 159-169. Schulz, T. C., Young, H. Y., Agulnick, A. D., Babin, M. J., Baetge, E. E., Bang, A. G., Bhoumik, A., Cepa, I., Cesario, R. M., Haakmeester, C., Kadoya, K., Kelly, J. R., Kerr, J., Martinson, L. A., McLean, A. B., Moorman, M. A., Payne, J. K., Richardson, M., Ross, K. G., Sherrer, E. S., Song, X., Wilson, A. Z., Brandon, E. P., Green, C. E., Kroon, E. J., Kelly, O. G., D'Amour, K. A. \& Robins, A. J. (2012). A Scalable system for production of functional pancreatic progenitors from human embryonic stem cells. PLOS One , 7 (5), e37004. Schulz, T. C. (2015). Concise Review: Manufacturing of pancreatic endoderm cells for clinical trials in type 1 diabetes. Stem Cells Transl. Med. 4 , 927-931. Smink, A. M., \& De Vos, P. (2018). Therapeutic strategies for modulating the extracellular matrix to improve pancreatic islet function and survival after transplantation. Curr Diab Rep. 18 , 39-46. Stendahl, J.C., Kaufman, D. B. \& Stupp, S. I. (2009). Extracellular matrix in pancreatic islets: Relevance to scaffold design and transplantation. Cell Trans . 18, 1-12. Takahashi, Y., Takebe, T. \& Taniguchi, H. (2016). Engineering pancreatic tissues from stem cells towards therapy. Regen. Ther.3 , 15-23. Tomov, M. L., Olmsted, Z. T., \& Paluh, J. L. (2015). The human embryonic body cystic core exhibits architectural complexity revealed by use of high throughput polymer microarrays. Macromol. Biosci,15 (7). Vegas, A. J., Veiseh, O., Gürtler, M., Millman, J.R., Pagliuca, F. W., Bader, A. R., Doloff, J. C., Li, J., Chen, M., Olejnik, K., Tam, H. H., 
Jhunjhunwala, S., Langan, E., Arestadasilva, S., Gandham, S., McGarrigle, J., Bochenek, M. A., Hollister-Lock, J., Obirholzer, J., Greiner, D. L., Weir, G. C., Melton, D. A., Langer, R. \& Anderson, D. G. (2016). Long term glycemic control using polymer encapsulated, human stemcell derived b-cells in immune competent mice. Nat Med ,22 (3), 306-311. Vieira, A., Courtney, M., Druelle, N., Avolio, F., Napolitano, T., Hadzic, B., Navarro-Sanz, S., Ben-Othman, N. \& Collombat, P. (2016). $\beta$-Cell replacement as a treatment for type 1 diabetes: an overview of possible cell sources and current axes of research. Diabetes, Obes. Metab. 18 , 137-143. Wang, Y., Hai, T., Liu, L. \& Zhou, Q. (2015). Cell therapy in diabetes: current progress and future prospects. Sci. Bull. 60(20) , 1744-1751. Wilmut, I., Leslie, S., Martin, N. G., Peschanski, M., Rao, M., Trounson, A., Turner, D., Turner, M. L., Yamanaka, S. \& Taylor, C. J. (2015). Development of a global network of induced pluripotent stem cell halpobank. Reg. Med . 10 (3), 235-238.

Tables

\begin{tabular}{lll}
\hline Scaffold & Modification & $\begin{array}{l}\text { Fold expansion in 10 days } \\
\text { (Mean } \pm \text { S.D. } \mathbf{N}=6)\end{array}$ \\
\hline U & Unmodified & $5.6 \pm 0.28$ \\
UG & Unmodified + Gelatin & $5.2 \pm 0.34$ \\
A & Amine-modified & $5.4 \pm 0.18$ \\
AG & Amine-modified + Gelatin & $4.6 \pm 0.32$ \\
N & NaOH-modified & $3.8 \pm 0.19$ \\
NG & NaOH-modified + Gelatin & $6.6 \pm 0.22$ \\
\hline
\end{tabular}

\section{FIGURE LEGENDS}

FIGURE 1. Pancreatic cell culture on cellulose scaffolds with chemical surface modifications. A) Cellulose scaffold modifications: unmodified (U), amine-modified (A) and $\mathrm{NaOH}$-modified $(\mathrm{N})$, without and with gelatin (G) coating. B) Cell seeding on cellulose scaffold showing phase image of pancreatic progenitors in dish (top), scanning electron microscopy image of cellulose scaffold prior to cell seeding with 20 to $50 \mu \mathrm{m}$ fibers (middle), and fluorescent image of DAPI-labeled cells on amine-modified scaffold (bottom). Scale bars are $50 \mu \mathrm{m}, 50 \mu \mathrm{m}$ and $20 \mu \mathrm{m}$ respectively. C) Schematic of multi-well analysis of variously modified cellulose scaffolds cut into $2 \times 3 \times 5 \mathrm{~mm}^{3}$ pieces. D) Schematic of wicking matrix bioreactor.

FIGURE 2. Viability and expansion of pancreatic cells on chemically modified cellulose scaffolds. A) Fluorescence microscopy images of pancreatic progenitors prior to seeding onto the cellulose scaffold. Cells are stained with antibodies against specific biomarkers of the pancreatic lineage and counterstained with DAPI (blue). Top panel scale bar $=50 \mu \mathrm{m}$, bottom panel scale bar $=10 \mu \mathrm{m}$. B) MTT assay of pancreatic cells on different surface-modified cellulose scaffolds during 10 days incubation.

FIGURE 3. Scanning electron microscopy (SEM) images of pancreatic cells on chemically modified cellulose scaffolds. Cells were maintained on the scaffolds and fixed at days 1, 3, 5 and 10 to evaluate cell attachment and growth throughout the scaffold. Black circles show cell aggregates on scaffold. Scale bar $=10 \mu \mathrm{m}$.

FIGURE 4. Differentiation of hiPSCs to pancreatic cells. A) Differentiation timeline. B) Phase images of differentiating hiPSCs at selected stages. Scale bar $=100 \mu \mathrm{m}$. C) Immunocytochemistry of differentiating cells at selected stages. Scale bar $=50 \mu \mathrm{m}$.

FIGURE 5. Viability and functionality of hiPSC-derived pancreatic cells on scaffold. A) MTT assay of pancreatic single cells (12 days) and aggregates (10 days) on amine-modified scaffolds. B) MTT assay of pancreatic single cells (12 days) and aggregates (10 days) on gelatin-coated, $\mathrm{NaOH-modified} \mathrm{scaffolds} \mathrm{C}$ ) Scanning electron microscopy of pancreatic single cells and aggregates seeded onto amine-modified scaffolds. 
Scale bar $=20 \mu \mathrm{m}$. C) Insulin release by pancreatic single cells (12 days) and aggregates (10 days) on amine-modified scaffolds. D) Insulin release by pancreatic single cells (12 days) and aggregates (10 days) on gelatin-coated, NaOH-modified scaffolds. E) Scanning electron microscopy of pancreatic single cells and aggregates seeded onto amine-modified scaffold. Scale bar $=20 \mu \mathrm{m}$. F) Scanning electron microscopy of pancreatic single cells and aggregates onto gelatin-coated, NaOH-modified scaffolds. Scale bar $=20 \mu \mathrm{m}$.

FIGURE 6. Growth and insulin secretion of pancreatic cells on amine-modified scaffolds in a miniature bioreactor. A) Bioreactor setup in the incubator. B) Scaffold incubated in MTT overnight at end of culture. Arrows indicate purple formazan crystals formed by viable cells on the scaffold. C) Scanning electron micrographs of pancreatic cells on the scaffold after 13 days in the bioreactor. Scale bar $=20 \mu \mathrm{m}$. D) Insulin secretion from pancreatic cells in the bioreactor during the 13-day culture period. Left panel, reactor seeded with $5 \times 10^{6}$ cells; right panel, reactor seeded with $1 \times 10^{7}$ cells. E) Metabolic profile of pancreatic cells seeded on the bioreactor during the 13-day culture period.

\section{Hosted file}

Figure1.pdf available at https://authorea.com/users/288981/articles/414728-insulinproduction-from-hipsc-derived-pancreatic-cells-in-a-novel-wicking-matrix-bioreactor

\section{Hosted file}

Figure2.pdf available at https://authorea.com/users/288981/articles/414728-insulinproduction-from-hipsc-derived-pancreatic-cells-in-a-novel-wicking-matrix-bioreactor

\section{Hosted file}

Figure3.pdf available at https://authorea.com/users/288981/articles/414728-insulinproduction-from-hipsc-derived-pancreatic-cells-in-a-novel-wicking-matrix-bioreactor

\section{Hosted file}

Figure4.pdf available at https://authorea.com/users/288981/articles/414728-insulinproduction-from-hipsc-derived-pancreatic-cells-in-a-novel-wicking-matrix-bioreactor

\section{Hosted file}

Figure5.pdf available at https://authorea.com/users/288981/articles/414728-insulinproduction-from-hipsc-derived-pancreatic-cells-in-a-novel-wicking-matrix-bioreactor

\section{Hosted file}

Figure6.pdf available at https://authorea.com/users/288981/articles/414728-insulinproduction-from-hipsc-derived-pancreatic-cells-in-a-novel-wicking-matrix-bioreactor 